\title{
Breast Care
}

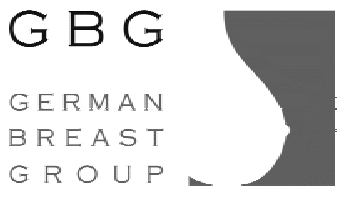

German Breast Group

PD Dr. Gunter v. Minckwitz

GBG Forschungs $\mathrm{GmbH}$

Schleussnerstr. 42, 63263 Neu-Isenburg

Tel. +49 102 79874-10

Fax +49 $10279874-40$

\section{Bericht vom 3. GBG-Jahrestreffen 03.02.-04.02.2006}

Mehr als 200 Ärztinnen und Ärzte sowie Studiendokumentare aus über 110 deutschen Kliniken und Instituten trafen sich am Freitag, den 03.02.2006 und Samstag, den 04.02.2006 im Hotel Lindner in Frankfurt am Main zum 3. Jahrestreffen der GBG Forschungs GmbH (German Breast Group). Die GBG Forschungs $\mathrm{GmbH}$ ist eine akademisch orientierte Forschungsorganisation (ARO), die im Jahr 2003 gegründet wurde. Sie ist eine Forschungseinrichtung zur Planung, Organisation und Durchführung nationaler und internationaler Studien zur Behandlung von Brustkrebs. Erst drei Jahre alt und weiterhin in Expansion begriffen demonstrierte die GBG auf dem Jahrestreffen ihre Führungsposition in der nationalen und internationalen Studienlandschaft. Ebenso wie die Zahl der an GBG-Studien teilnehmenden Kliniken und Institute innerhalb des letzten Jahres von 246 auf 413 anstieg, hat sich die Anzahl an rekrutierten Patienten 2005 von 1085 auf 2190 verdoppelt. Zurzeit betreut die GBG 11 laufende Studien, die den kompletten Bereich der Therapie von Brustkrebs, angefangen bei der Prävention über Neoadjuvanz und Adjuvanz bis hin zur Palliation abdecken. Diese Studien werden zum Teil in Kooperation mit der AGO, NOGGO und WSG durchgeführt.

Das Anliegen des diesjährigen Jahrestreffens war es einerseits, die laufenden GBG-Studien nochmals detailliert vorzustellen, andererseits den Teilnehmern ein Podium für Fragen und die Diskussion von Problemen zu geben. In der neoadjuvanten Situation ist besonders die GeparQuattro-Studie zu erwähnen, die durch die Integration von Capecitabin in das üblicherweise angewendete Regime aus Antrazyklin und Taxan, bzw. durch die Verlängerung der Behandlung ( 8 vs. 12 Zyklen) ein Ansprechen auf die primär systemische Therapie verbessert möchte. In der adjuvanten Situation bietet die GAIN-
Studie mit einem dosisintensivierten und dosisdichten Regime ein Therapiekonzept für Patienten der Hochrisikogruppe, die ICE-Studie wiederum gibt Behandlungsmöglichkeiten für die ältere an Brustkrebs erkrankte Frau. Auch die NATAN (neoadjuvant trial add on study) und ZORO-Studie (Zoladex rescue of ovarian function) bieten innovative Therapiekonzepte für Patienten in der Adjuvanz an und tragen dazu bei, auf nationalem sowie internationalem Gebiet die Behandlung von Brustkrebspatienten zu optimieren. Auch kleinere Studien wie zum Beispiel Präventions- (IBIS II) und Palliativstudien (Monica, Rita, TBP) bieten moderne Möglichkeiten der Vorbeugung und Behandlung von Brustkrebs an. Wie erfolgreich und effektiv die bereits abgeschlossenen Studien der GBG waren, zeigt sich in vielen Veröffentlichungen in international renommierten Fachzeitschriften sowie der Präsentationen von Studienergebnissen auf nationalen und internationalen Kongressen. Des Weiteren konnten auf dem Jahrestreffen auch neue Therapieoptionen vorgestellt werden, die die GBG im Jahr 2006 verfolgen wird. Die Übernahme der großen NNBC-3 Studie für nodal negative Brustkrebspatientinnen als Sponsor wird im Frühling 2006 erfolgen und damit auch dieser großen Patientengruppe die Teilnahme in einem Studienkonzept zur Therapie von Brustkrebs ermöglichen. Zwei weitere Phase-II-Studien in der palliativen Situation sind mit dem Tyrosinkinaseinhibitoren Lapatinib als auch dem mTOR-Inhibitor RAD001 in Arbeit und werden Mitte 2006 starten.

Verschiedene Workshops betonten während des zweitägigen Jahrestreffens die praktische Komponente der Arbeit in klinischen Studien. Hier wurde sowohl auf rechtliche Aspekte bei der Planung und Durchführung von Studien eingegangen, als auch Studiendokumentationen an prägnanten Beispielen erarbeitet und besprochen. Wichtige Themen aus dem klinischen Alltag wie die Kommunikation mit Tumorpatienten wurden vertieft und konnten den Teilnehmern so manchen praktischen Tipp mitgeben. Ein ausführlicher Vortrag über das Management von Nebenwirkungen unter Chemotherapien rundete das gelungene Konzept des GBG-Jahrestreffens ab. Alle Vorträge des GBG-Jahrestreffens sind auf der Internetseite www.germanbreastgroup.de zu erhalten.

Die gelungene Mischung aus wissenschaftlichen Vorträgen, Workshops und regen Diskussionen zeigt, dass wir uns in Deutschland auf dem richtigen Weg zu objektiven, unabhängigen und akademisch professionellen Studien befinden, denn nur auf diese Weise gewonnene, qualitativ hochwertige Studienergebnisse sind beständig genug, um wirklich zu einer Verbesserung der Brustkrebstherapie zu führen.

Dr. med. S. Vescia, GBG 